\title{
Patterns of Volunteer Behaviour Across Online Citizen Science
}

\author{
Helen Spiers ${ }^{1}$, Alexandra Swanson ${ }^{2}$, Lucy Fortson ${ }^{3}$, Brooke D. Simmons ${ }^{4}$, \\ Laura Trouille ${ }^{5}$, Samantha Blickhan ${ }^{6}$, Chris Lintott ${ }^{7}$ \\ ${ }^{1,2,7}$ Department of Physics, University of Oxford; ${ }^{3}$ School of Physics and Astronomy, University of Minnesota; ${ }^{4}$ Center for \\ Astrophysics and Space Sciences, University of California; ${ }^{5,6}$ Adler Planetarium, Chicago \\ \{helen, ali, lucy, brooke, trouille, samantha\}@zooniverse.org, cjl@astro.ox.ac.uk
}

\begin{abstract}
Human-computer systems are increasingly applied to data reduction problems; citizen science platforms (e.g. the Zooniverse) are one type of such a system. These platforms function as social machines, combining volunteer efforts with automated processes to enable distributed data analysis. The rapid growth of this approach is increasing the need to understand how we can improve volunteer interaction and engagement. Here, we utilize the most comprehensive collection of online citizen science data gathered to date to examine multiple variables across 63 Zooniverse projects. Our analyses reveal how subtle design changes can influence many facets of volunteer interaction, generating insights that have implications for the design and study of citizen science projects, and future research.
\end{abstract}

\section{KEYWORDS}

Citizen Science; Volunteer Behaviour; Project Design

\section{INTRODUCTION}

The unprecedented volumes of data being produced across multiple knowledge domains provide an incredible opportunity to advance understanding, but also represent a growing analytical challenge, as data complexity frequently outstrips computer-based analytical methods. One innovative solution that is gaining in popularity is to perform distributed data analysis through applying human pattern recognition abilities via online citizen science platforms [1].

However, the exponential growth in project number is creating a need to improve understanding of volunteer patterns of behaviour, as the sustainability of this approach is contingent on continued volunteer interaction. Here, we utilize the Zooniverse platform as a 'web observatory ecosystem' to examine patterns of volunteer behaviour across $n=63$ projects. The analyses we present here provide quantitative evidence informative to researchers leading online citizen science projects, and are of utility to researchers examining crowdbased knowledge production.

\section{METHODOLOGY}

Data for $\mathrm{n}=63$ projects were obtained from Zooniverse databases. To examine volunteer demographic features data were extracted

This paper is published under the Creative Commons Attribution-NonCommercial-NoDerivs 4.0 International (CC BY 4.0) license. Authors reserve their rights to disseminate the work on their personal and corporate Web sites with the appropriate attribution.

WWW'18 Companion April 23-27, 2018, Lyon, France.

(c) 2018 IW3C2 (International World Wide Web Conference Committee), published under Creative Commons CC BY 4.0 License.

ACM ISBN 978-1-4503-5640-4/18/04.

DOI: https://doi.org/10.1145/3184558.3186945 from Google Analytics for the five most recently launched astronomy and ecology projects, from January $1^{\text {st }} 2017$ to March $31^{\text {st }}$ 2017 (data obtained May $9^{\text {th }} 2017$ ).

\section{RESULTS}

\subsection{How heterogeneous is classification and volunteer activity across projects?}

Notable heterogeneity is observed among Zooniverse projects for the total number of classifications received within the first 100 days post-launch, with three orders of magnitude difference between the project with the most classifications (Space Warps; n classifications $=8,011,730$ ) versus the project with the fewest (Microplants; $n$ classifications $=8,577$ ), indicating project inclusion on a successful citizen science platform does not guarantee high levels of interaction.

Examining temporal trends in classification activity revealed that the majority of Zooniverse projects have a similar classification curve characterized by a peak of activity upon launch that rapidly declines (Figure 1a). Of the projects assessed, Supernova Hunters showed striking exception to this and instead displayed a recurring peak of activity each week (Figure 1b), arising from the regular release of new project data and concurrent e-newsletter notification to project volunteers [2]. Notably, volunteer classification activity on the Supernova Hunters project has begun to precede e-newsletter mailings, indicating that volunteers anticipate data release and are deeply engaged. Based on this finding, it would seem reasonable to recommend other projects adopt a similar format of incremental data release and frequent volunteer interaction to garner more consistent engagement, but, as shall be considered below, adopting such an approach may have unintended consequences.

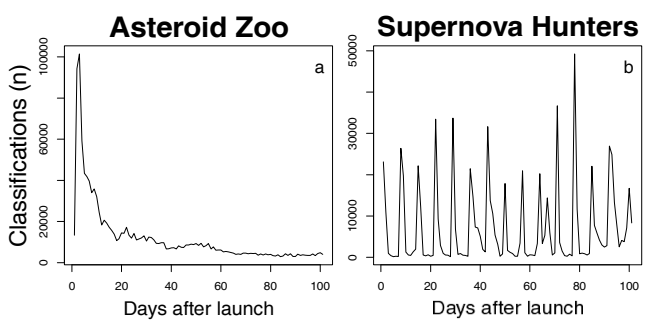

Figure 1: Supernova Hunters has a distinctive classification curve.

\subsection{How skewed are volunteer contributions in Zooniverse projects?}

Previous analyses have found skewed distributions of volunteer contributions in Zooniverse projects [3,4]. Here, we sought to 
extend these analyses by examining a larger number of projects. Using an approach often used to study income inequality [5], we plotted the Lorenz curve for the distribution of volunteers' total classifications for each project (Figure 2a) and calculated corresponding Gini coefficients. For all projects we observe a large area between the Lorenz curve and the $45^{\circ}$ line (perfect equality), indicating that across projects, a large fraction of classifications are provided by a relatively small number of volunteers. However, heterogeneity in volunteer contribution equality was observed between projects - with Gini coefficients ranging from 0.94 for Supernova Hunters (Figure 2b) to 0.54 for Microscopy Masters (Figure 2c).

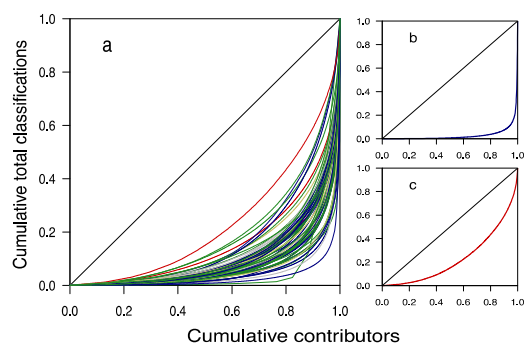

Figure 2: All Zooniverse projects display unequal volunteer classification contribution.

\subsection{Do projects associate with different volunteer demographics?}

We next described demographic features of volunteers contributing to Zooniverse projects from differing domains (see Methods). Examining classification page views subset by visitor age revealed no consistent trend across the astronomy (Figure 3a) or ecology projects (Figure 3b), suggesting Zooniverse projects appeal to a broad age-range. The Supernova Hunters project was notably popular amongst the oldest age group (65+ years old). In contrast, striking sex differences were observed, with astronomy projects showing clear male bias (Figure 3c) and ecology projects showing greater variability in their sex balance (Figure 3d).
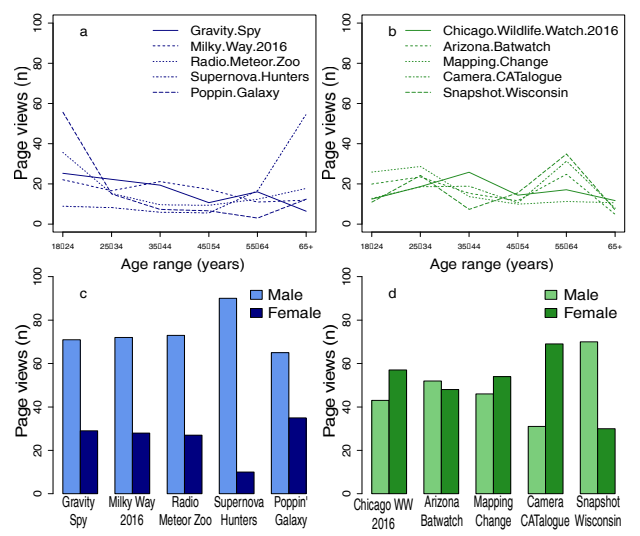

Figure 3: Domain-specific demographic features are observed for Zooniverse projects.

\section{DISCUSSION}

Here we analyse multiple variables from the most comprehensive collection of online citizen science project data gathered to date. Key findings include that, despite high levels of heterogeneity in total number of classifications, project classification curves were highly comparable with one striking exception: the Supernova Hunters project. This project displayed weekly peaks of activity, reflecting the scheduled release of small amounts of new data, suggesting data scarcity drives increased project engagement. Researchers leading citizen science projects may consider adopting a similar study design. However, this may have effects beyond encouraging more frequent volunteer participation - we found Supernova Hunters to be the most unequal project in terms of volunteer classification contributions - the majority of classifications in this project are made by a small cohort of highly dedicated volunteers.

Clear benefits exist in developing a small community of highly engaged and well-practised volunteers who regularly return to a project at the time of data release, as this allows for faster and more accurate processing of data. However, our analyses show that this makes the volunteer community contributing to a particular project less diverse. We found that the Supernova Hunters community is demographically biased towards men of retirement age. It is possible that the design of this project has excluded those unable to offer a regular, weekly time-commitment during the working day, and it may be the project appeals more to older males, or less to other demographics, due to the increased competition or due to the higher science capital perceived to be required to contribute.

Cultivating the selection of a small, highly dedicated community of volunteers may benefit challenging projects that require a high level of skill, however, this may be deleterious for other project types; for example, a health-related project may benefit from a more representative cross-section of volunteers. It should also be considered whether this is acceptable to the broader ethos of citizen science, as frequently project goals extend beyond productivity to include contributing to 'social good'. Is it acceptable to the practice of citizen science to cultivate scenarios that intentionally restrict the opportunity of the full volunteer community to access a project?

Project design can influence many facets of the nature of volunteer participation, including who participates, when and how much. Whether a particular design choice is beneficial or harmful to a particular project should be considered carefully in relation to project goals.

\section{REFERENCES}

[1] Bonney R, et al. (2009) Citizen Science: A Developing Tool for Expanding Science Knowled and Scientific Literacy. BioScience 59(11):977-984

[2] Wright DE, et al. (2017) A transient search using combined human and machine classificatiou Monthly Notices of the Royal Astronomical Society 472(2):1315-1323.

[3] Sauermann H \& Franzoni C (2015) Crowd science user contribution patterns and th implications. Proc Natl Acad Sci 112(3):679-684.

[4] Cox J (2015) How is success defined and measured in online citizen science? A case study Zooniverse projects. Computing in Science and Engineering, 17 (4): 28-41.

[5] Gastwirth JL (1972) The Estimation of the Lorenz Curve and Gini Index. Rev Econ S 54(3):306-316. 